\title{
RESEARCH
}

Open Access

\section{Three-dimensional cultured mesenchymal stem cells enhance repair of ischemic stroke through inhibition of microglia}

Yuejiao Li ${ }^{1,2 \dagger}$, Yankai Dong ${ }^{2,1 \dagger}$, Ye Ran ${ }^{3 \dagger}$, Yanan Zhang ${ }^{1,4}$, Boyao Wu', Jundong Xie ${ }^{1,4}$, Yanpei Cao ${ }^{5}$, Miaohua Mo ${ }^{4}$, Sen $\mathrm{Li}^{1}$, Hao Deng ${ }^{6}$, Wenbo $\mathrm{HaO}^{6}$, Shengyuan $\mathrm{Yu}^{7^{*}}$ and Yaojiong $\mathrm{Wu}^{1,4^{*}}$ (D)

\begin{abstract}
Background: We show previously that three-dimensional (3D) spheroid cultured mesenchymal stem cells (MSCs) exhibit reduced cell size thus devoid of lung entrapment following intravenous (IV) infusion. In this study, we determined the therapeutic effect of 3D-cultured MSCs on ischemic stroke and investigated the mechanisms involved.
\end{abstract}

Methods: Rats underwent middle cerebral artery occlusion (MCAO) and reperfusion. $1 \times 10^{6}$ of 3D- or 2D-cultured MSCs, which were pre-labeled with GFP, were injected through the tail vain three and seven days after MCAO. Two days after infusion, MSC engraftment into the ischemic brain tissues was assessed by histological analysis for GFPexpressing cells, and infarct volume was determined by MRI. Microglia in the lesion were sorted and subjected to gene expressional analysis by RNA-seq.

Results: We found that infusion of 3D-cultured MSCs significantly reduced the infarct volume of the brain with increased engraftment of the cells into the ischemic tissue, compared to 2D-cultured MSCs. Accordingly, in the brain lesion of 3D MSC-treated animals, there were significantly reduced numbers of amoeboid microglia and decreased levels of proinflammatory cytokines, indicating attenuated activation of the microglia. RNA-seq of microglia derived from the lesions suggested that 3D-cultured MSCs decreased the response of microglia to the ischemic insult. Interestingly, we observed a decreased expression of mincle, a damage-associated molecular patterns (DAMPs) receptor, which induces the production of proinflammatory cytokines, suggestive of a potential mechanism in 3D MSC-mediated enhanced repair to ischemic stroke.

Conclusions: Our data indicate that 3D-cultured MSCs exhibit enhanced repair to ischemic stroke, probably through a suppression to ischemia-induced microglial activation.

Keywords: Mesenchymal stem cells, Ischemic stroke, Microglia, Mincle, Neuroinflammation

\footnotetext{
* Correspondence: yusy1963@126.com; wu.yaojiong@sz.tsinghua.edu.cn

†Yuejiao Li, Yankai Dong and Ye Ran contributed equally to this work.

${ }^{7}$ Department of Neurology, The Chinese PLA General Hospital, Beijing

100853, China

'State Key Laboratory of Chemical Oncogenomics, and Shenzhen Key

Laboratory of Health Sciences and Technology, Tsinghua Shenzhen

International Graduate School, Tsinghua University, Shenzhen 518055, China

Full list of author information is available at the end of the article
}

(C) The Author(s). 2021 Open Access This article is licensed under a Creative Commons Attribution 4.0 International License, which permits use, sharing, adaptation, distribution and reproduction in any medium or format, as long as you give appropriate credit to the original author(s) and the source, provide a link to the Creative Commons licence, and indicate if changes were made. The images or other third party material in this article are included in the article's Creative Commons licence, unless indicated otherwise in a credit line to the material. If material is not included in the article's Creative Commons licence and your intended use is not permitted by statutory regulation or exceeds the permitted use, you will need to obtain permission directly from the copyright holder. To view a copy of this licence, visit http://creativecommons.org/licenses/by/4.0/. The Creative Commons Public Domain Dedication waiver (http://creativecommons.org/publicdomain/zero/1.0/) applies to the data made available in this article, unless otherwise stated in a credit line to the data. 


\section{Background}

Ischemic stroke is the first leading cause of death and neurological dysfunction in China [1]. Currently, only intravenous thrombolysis with tissue plasminogen activator and mechanical thrombectomy have shown to be effective in the acute phase of ischemic stroke. However, these approaches are not promptly accessible to most patients due to a narrow therapeutic window [2]. There has been no effective drug to ameliorate sensorimotor functions except for rehabilitation, leaving most of the patients suffering from life-long disability. In this sense, cell therapy has emerged as a promising therapy for stroke in the subacute phase. Mesenchymal stem cells (MSCs) have attracted much attention due to advantages over other types of cells, including absence of ethical and legal concerns, low immunogenicity, and sufficient availability [3-5]. In preclinical and clinical studies, the non-invasive intravenous (IV) route is the most commonly used approach for MSCs delivery [6]. Many literatures have shown that IV administration of MSCs promoted tissue repair and had a therapeutic effect on ischemic stroke because of its multiple differentiation potential, anti-inflammatory and immunomodulatory properties, and expression of trophic factors and cytokines [7-9]. However, the loss of homing and engraftment potential in cultured MSCs after expanding under conventional two-dimensional (2D) culture conditions has warranted the development of novel clinical strategies [10]. Therefore, efforts need to be made to improve the homing ability of culture expanded MSCs after cell transplantation.

Our and others' previous studies have shown that three-dimensional (3D) culture significantly reduced the size of MSCs compared with 2D monolayer culture, thus reducing the vascular obstructions in the lungs after IV injection [11-15]. Besides, compared with 2D MSCs, 3D MSCs exhibited decreased expression of integrins, as excess expression of integrins after 2D culture is a cause for MSC entrapment in the lung [15]. The escape of MSCs from the lung allows the cells to circulate in the blood and therefore distribute more efficiently into other tissues [13, 15]. Furthermore, studies have shown that the expression of homing receptors, such as CXCR4, is usually decreased on the surface of culture-expanded MSCs [16]. 3D culture restores the expression levels of CXCR4 in MSCs and improves their homing capacity to the injury sites after systematic administration [13, 16-18].

It has been suggested that inflammation plays an important role in the pathogenesis of ischemic stroke [19]. As resident macrophage cells, microglia are the principal immune cells of the brain, and the first to respond to the pathophysiological changes induced by ischemic stroke [19]. In the early stages of stroke, classically activated microglia contribute to neuronal damage and exacerbate tissue injury by producing inflammatory cytokines and cytotoxic substances [20]. In vivo studies have shown that MSC administration significantly reduced microglia activation, as indicated by decreased immunoreactivity of ED1 and ionized calcium-binding adapter molecule 1 (Iba1) [21]. Pre-conditioning of MSCs under 3D environments prior to transplantation increased their anti-inflammatory properties by secreting more immunoregulatory factors such as prostaglandin E2, transforming growth factor beta 1, interleukin-6 (IL-6), tumor necrosis factor-stimulated gene-6 (TSG-6), hepatocyte growth factor (HGF), and stanniocalcin 1 (STC1) [13, 22-25]. In vitro study has demonstrated that 3D MSCs reduced the macrophages activation and promoted the transition of stimulated macrophages from a proinflammatory M1 phenotype to a more anti-inflammatory phenotype [13, 23]. Another study showed a decreased infiltration of macrophages with 3D-exosomes treatment in the cisplatin-induced murine acute kidney injury model [26]. However, the immunoregulatory effects of 3D MSCs in the ischemic stroke animal model are not elucidated, especially on the modulation of microglia.

In this study, we found that after IV infusion, 3D MSCs exhibited enhanced homing ability to the ischemic lesion, reduced the infarct volume, and restored the neurological dysfunctions in rats with ischemic stroke, compared to 2D MSCs. In addition, 3D MSC treatment reduced the activation of microglia with decreased immunoreactivity of Iba1.

\section{Methods}

\section{Animals}

Male Sprague-Dawley rats, average weighing 280-320 g were obtained from the Laboratory Animal Center, Guangdong Province, China. All of the animals were maintained in cages at a temperature-controlled environment, with a 12-h light-dark cycle and free access to food and fresh water. The procedures involving animal and their care were conducted under the approval of the Ethics Committee of Tsinghua University.

\section{Middle cerebral artery occlusion model}

Middle cerebral artery occlusion (MCAO) was induced in male Sprague-Dawley rats as described in our previous studies [27]. Briefly, animals were anesthetized with a mixture of 1 to $2 \%$ isoflurane in nitric oxide/oxygen via a face mask. The body temperature was maintained at $37{ }^{\circ} \mathrm{C}$ during the surgical procedures. A midline cervical incision was made with subsequent exposure of the left common carotid artery, internal carotid artery, and external carotid artery. The common carotid artery and external carotid artery were ligated with a 4-0 silk. Thereafter, a silicon-rubber-coated round-tip nylon 
suture was advanced from the common carotid artery until it blocked the origin of the middle cerebral artery. After 120 min of MCAO, animals were reanesthetized and reperfused by withdrawing the nylon suture.

\section{Cell culture}

MSCs were isolated from human placenta as previously described [28]. Cells were culture expanded in Dulbecco's modified Eagle's medium (DMEM; Corning) supplemented with $10 \%$ fetal bovine serum (FBS; Biological Industries) at $37{ }^{\circ} \mathrm{C}$ with $5 \% \mathrm{CO}_{2}$. Spheroid culture of MSCs were performed by culturing MSCs in cell culture spinner flasks in Minimum Essential Medium Eagle (MEM, Sigma) supplemented with 5\% EliteGro ${ }^{\mathrm{Tm}}-\mathrm{Adv}$ (Biomedical EliteCell Corp.) for $60 \mathrm{~h}$ at $37{ }^{\circ} \mathrm{C}$ with $5 \%$ $\mathrm{CO}_{2}$. To obtain single cells from spheroids, spheroids were incubated with $0.25 \%$ trypsin/EDTA at $37{ }^{\circ} \mathrm{C}$ for 4-6 min with gentle pipetting every $2-3 \mathrm{~min}$.

\section{Intravenous administration of MSCs}

The animals were randomly divided into the following three experimental groups after MCAO: PBS, 2D MSCs, and 3D MSCs. In one set of experiments, the rats were slowly infused with $1 \times 10^{6}$ cells/200 $\mu \mathrm{l}$ PBS into the tail vein at 3 days and 7 days after MCAO. The same amount of PBS was injected as the control. The animals were sacrificed at 2 weeks after stroke. In another set of experiments, $3 \times 10^{6} 2 \mathrm{D}$ or $3 \mathrm{D}$ MSCs in $200 \mu \mathrm{l}$ PBS were intravenously injected 3 days after MCAO as mentioned above. The rats were sacrificed at 2 days after cell transplantation. To investigate the viability and engraftment of MSCs after infusion, cells were labeled using a green fluorescent protein (GFP) lentivirus (Cyagen) according to the manufacturer's instructions. Cells were filtered through a $40-\mu \mathrm{m}$ cell strainer to generate a single cell suspension before transplantation.

\section{Behavioral testing}

The behavioral tests were performed with modified neurological severity score (mNSS) and adhesiveremoval test at $1,5,9$, and 13 days post-MCAO as previously described $[29,30]$. mNSS test was used to evaluate the neuromotoric capacity of the animals. Motor, sensory, balance, and reflex tests are included and are graded on a scale of 0 to 18 ( 0 for normal score; 18 for maximal deficit score). For adhesiveremoval tests, round dots of adhesive tape were used as bilateral tactile stimuli, occupying the distal-radial region of each forelimb wrist. The time, to a maximum of $120 \mathrm{~s}$, for each rat to remove the right paper dots was recorded. Three trials were conducted daily, with an interval of at least five minutes. Data are presented as absolute values in seconds (adhesiveremoval test) or score points (mNSS test).

\section{Immunofluorescence}

Rats were anesthetized with sodium pentobarbital (30 $\mathrm{mg} / \mathrm{kg}$, i.p.) and perfused with $100 \mathrm{~mL}$ saline and 100 $\mathrm{mL} 4 \%$ PFA. Tissues were dissected and fixed in 4\% PFA at $4{ }^{\circ} \mathrm{C}$ overnight. Fixed samples were dehydrated with $10 \%, 20 \%$, and $30 \%$ sucrose successively and embedded in OCT. Frozen sections of $10-\mu \mathrm{m}$ thickness were cut through the entire forebrain. Several consecutive sections of the entire brain were selected and processed for immunofluorescent staining with the appropriate primary antibody at $4{ }^{\circ} \mathrm{C}$ overnight against NeuN (a neuronal marker; Millipore, MAB377; 1:100), GFAP (a marker of astrocytes; Cell Signaling Technology, 12389; 1:200), and Iba1 (a microglia marker; Abcam, ab178846; 1:200). Matching fluorescence-conjugated secondary antibodies from Jackson ImmunoResearch were employed. Nuclei were counterstained with DAPI. All samples were analyzed with a confocal laser scanning microscope (FV1000, Olympus, Japan).

\section{Quantitative reverse transcription polymerase chain reaction (qRT-PCR) analysis}

Total RNA was extracted from the fresh brain tissues and MSCs using the RNAiso Plus reagent (Takara, 9109), according to the manufacturer's instructions. RNA was quantified using Nanodrop (Thermo Scientific). cDNA was synthesized from $2 \mu \mathrm{g}$ of total RNA with Hifair II 1st Strand cDNA Synthesis SuperMix for qPCR (Yeasen, 11123ES60). Next, qRT-PCR was performed on 7300 Real Time PCR System (Applied Biosystems) using Hieff qPCR SYBR Green Master Mix (High Rox Plus, Yeasen, 11203ES08) in triplicate. The relative expression of target genes was analyzed according to the $2^{-\triangle \Delta C T}$ method and normalized to the level of GAPDH. All primer sequences were shown in Supplementary Table S1.

\section{Fluorescence-activated cell sorting}

At 2 days after cell infusion, rats were deeply anesthetized with sodium pentobarbital (30 $\mathrm{mg} / \mathrm{kg}$, i.p.) and subjected to transcardial perfusion with $200 \mathrm{~mL}$ sterile ice-cold saline. After decapitation, each rat brain was harvested and the diseased hemispheres were stored in HBSS (without calcium, magnesium) (Thermo Fisher Scientific, C14175500BT). Isolated brain tissues were sliced into small pieces using a sterile scalpel on ice and enzymatically homogenized at $30{ }^{\circ} \mathrm{C}$ for 30 min with a pre-heated enzyme mix containing papain (100 U, Worthington, LS003119), dispase II (6 U, Sigma-Aldrich D4693), and DNAse I (100 U, Sigma-Aldrich D5025). Prior to use, papain was activated for $30 \mathrm{~min}$ at $37{ }^{\circ} \mathrm{C}$ and $5 \% \mathrm{CO}_{2}$. To stop all digestions, samples were diluted with cold HBSS and placed on ice. Solutions were then further homogenated using Pasteur pipette with the 
fire-polished tip for 10 times in approximately $45 \mathrm{~s}$ and filtered through a $70-\mu \mathrm{m}$ cell strainer (BD, 352350) to remove large debris and cell clusters. The resulting single cell suspension was centrifuged at $300 \mathrm{~g}$ for $10 \mathrm{~min}$ at RT. Myelin and debris were removed using Percoll (Yeasen, 40501ES60) density gradient centrifugation. Briefly, a single suspension of cells was mixed with $9 \mathrm{~mL}$ of $30 \%$ isotonic Percoll solution and centrifugated at $300 \times g$ for $30 \mathrm{~min}$, at $18{ }^{\circ} \mathrm{C}$. After centrifugation, cells were collected from the bottom layer of the $30 \%$ Percoll gradient, washed by adding $1 \times$ HBSS and centrifugated for $10 \mathrm{~min}$ at $300 \times \mathrm{g}$. The cells were resuspended with 1 mL DMEM. Following this, the resuspended cells were stained with trypan blue and cell counting was performed using a hemocytometer. Cells were pre-blocked with excess irrelevant purified Ig from same isotype as the antibodies used for immunofluorescent staining on ice for $10 \mathrm{~min}$. Then, the cells were labeled for surface markers CD45 PE (202207, 1:100, BioLegend) and CD11b APC (201809, 1:100, BioLegend) in Cell Staining Buffer (420201, Biolegend) on ice for $30 \mathrm{~min}$ in the dark. Dead cells were excluded from analysis by labeling with 7-AAD Viability Staining Solution (40745ES64, Yeasen). $\mathrm{CD} 45^{\text {low }} \mathrm{CD} 11 \mathrm{~b}^{+}$microglia were sorted on SH800S Cell Sorter (Sony Biotechnology) and the data was analyzed with FlowJo software (Tree Star).

\section{RNA-sequencing}

Total RNA was extracted from the isolated microglia from the brain using TRIzol reagent. Subsequently, the total RNA was identified and the full-length cDNA was amplified by PCR. The average molecule length was determined using the Agilent 2100 bioanalyzer instrument (Agilent High Sensitivity DNA Reagents). Library was constructed following Tagmentation-based library construction protocol. PCR products were purified and selected with the Agencourt AMPure XP-Medium kit. DNA was quantified by Agilent Technologies 2100 bioanalyzer. Library was qualified by the Agilent Technologies 2100 bioanalyzer. The library was amplified to make DNA nanoball (DNB) which had more than 300 copies of one molecular. The DNBs were loaded into the patterned nanoarray and single end 50 bases reads were generated in the way of sequenced by combinatorial Probe-Anchor Synthesis (cPAS). The raw sequencing data were aligned to the rat genome. Differentially expressed genes (DEGs) were identified by PossionDis analysis with FDR $<0.001$.

\section{Microglial cell culture and activation}

The BV2 mouse microglial cell line was bought from China Center for Type Culture Collection. Cells were culture expanded at $37{ }^{\circ} \mathrm{C}$ with $5 \% \mathrm{CO}_{2}$ in DMEM (Corning, 10-017-CV) supplemented with 10\% FBS
(Biological Industries) and antibiotics. To assess the effects of 2D and 3D MSCs on BV2 cells, a total of $3 \times$ $10^{5} \mathrm{BV} 2$ cells were placed in a 6-well chamber and stimulated with $100 \mathrm{ng} / \mathrm{ml}$ LPS for $4 \mathrm{~h}$. One hundred microliters of conditioned medium from 2D or 3D MSCs were added simultaneously. After $4 \mathrm{~h}$ of treatment, BV2 cells were harvested for RNA extraction.

\section{Statistical analysis}

Statistical analysis of all experiments was carried out using GraphPad Prism 8.0 software. All data were presented as means \pm SEM. Unpaired Student's $t$ tests were performed to compare differences between two groups. One-way analysis of variance was used to compare differences involving three or more groups. $P$ value less than 0.05 was considered statistically significant.

\section{Results}

3D spheroid culture reduces the size of MSCs

After 3D spheroids culture, the diameter of MSCs significantly decreased (Fig. 1A-D), which is consistent with our previous studies [11, 14, 27]. Importantly, annexin V/PI staining of the cells by flow cytometry analysis showed that the 3D spheroid culture did not increase cell apoptosis (Fig. 1E, F). This indicates that the 3D spheroid culture can reduce the size of MSCs without affecting cell viability.

\section{D MSC infusion decreases infarct volume and improves neurological function}

In order to investigate the effects of $2 \mathrm{D}$ and 3D MSCs on the recovery of ischemic injury, MCAO rats were intravenously injected with PBS, $1 \times 10^{6}$ of $2 \mathrm{D}$ or $3 \mathrm{D}$ MSCs at 3 and 7 days after MCAO, respectively (Fig. 2A). The lesion volume measured by MRI at 16 days poststroke in 3D MSC-treated rats was significantly lower than that in the PBS group and the 2D MSCs group (Fig. 2B, C). Behavioral improvements were monitored on 1, 5, 9, and 13 days following MCAO. Neurological function was tested by mNSS before and after MSC treatment, demonstrating that injection of 3D MSCs significantly improved functional recovery compared to that of the PBS group at 5, 9, and 13 days after stroke. Whereas the neurological function in 2D MSC-treated rats was indistinguishable from the PBS group. To evaluate forelimb deficits, the impaired forelimb usage was further analyzed via the adhesive-removal test. Results showed that rats treated with 3D MSCs removed the tape significantly faster than rats treated with PBS (Fig. 2D). Together, the mNSS and the adhesive-removal test suggest that IV administration of 3D MSCs enhances sensorimotor outcomes after ischemic injury. To explain whether these functional improvements are due to neuronal survival, the expression of neuronal marker 

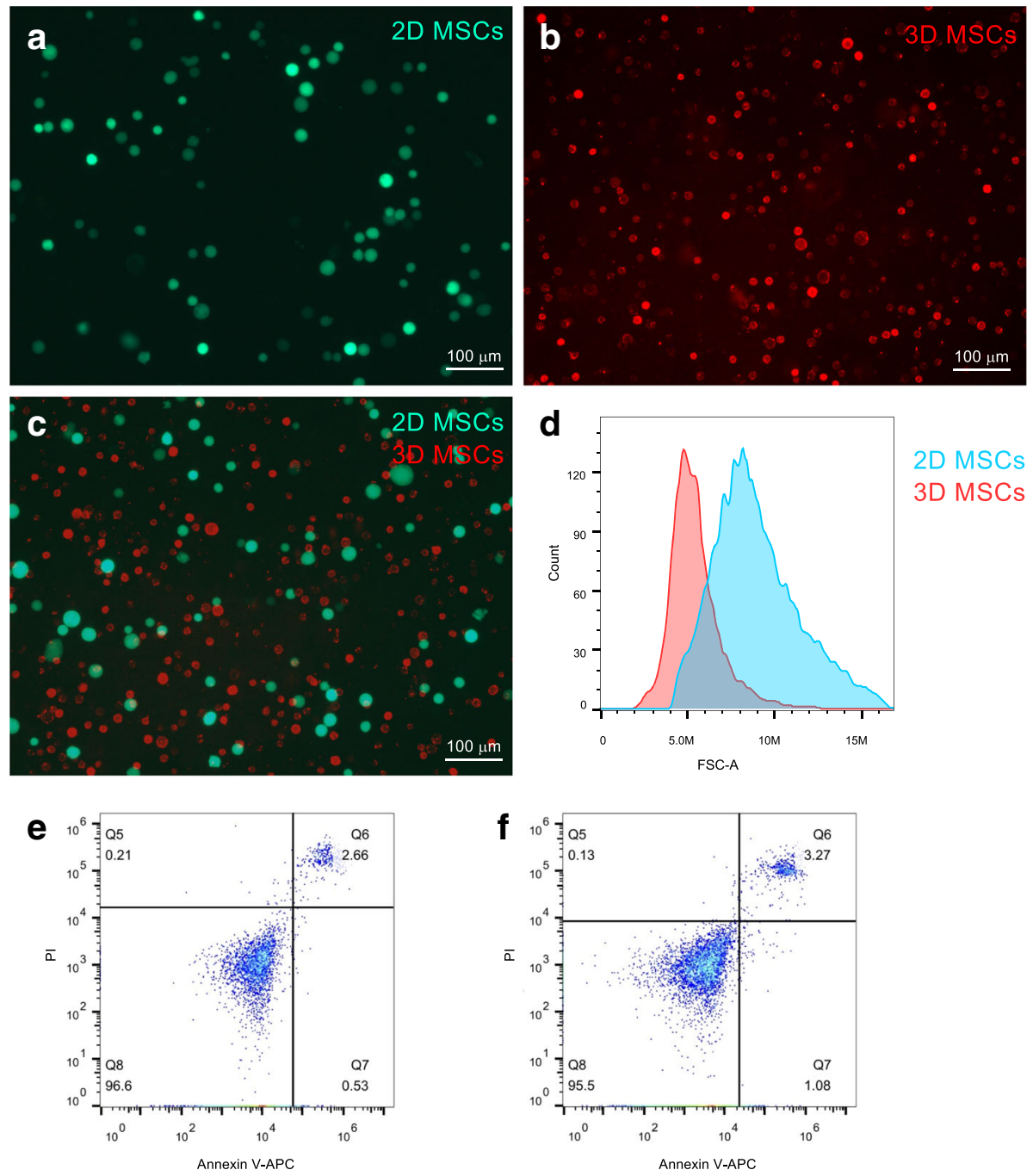

Fig. 1 Three-dimensional (3D) spheroid culture reduced the size of mesenchymal stem cells (MSCs). Two-dimensional (2D) MSCs a and 3D MSCs b were labeled with GFP and Dil, respectively. Photos showed the size of MSCs. c Comparison of the size of 2D and 3D MSCs. d Histogram showed the size of 2D and 3D MSCs with flow cytometry. The apoptosis of 2D MSCs (e) and 3D MSCs (f) were analyzed with annexin V/PI staining

NeuN was additionally determined using immunofluorescence imaging at 5 days after MCAO. Results showed that the number of NeuN-positive neurons was significantly increased in 3D MSC-treated animals compared to the PBS- and 2D MSC-treated rats (Fig. 2E, F). These data demonstrated that systematic administration of 3Dcultured MSCs significantly improved the ischemic brain repair.

\section{D MSCs exhibits enhanced homing ability to the brain} As 3D culture was reported to restore the expression of homing receptors of MSCs [13], we determined the homing of 3D MSCs to the ischemic lesion with immunofluorescence imaging. Both GFP-labeled 2D and 3D MSCs were observed in the ischemic hemisphere at
2 days after infusion, whereas significantly more 3D MSCs were detected than 2D MSCs (Fig. 3A, B). Besides, compared with the contralateral hemisphere, most MSCs were observed in the ischemic hemisphere (Fig. 3C). These results suggest that 3D-cultured MSCs improved the neurological repair partly through their enhanced homing ability to the ischemic lesion.

\section{D MSCs ameliorate ischemia-induced microglial activation in vivo}

Spheroid culture of MSCs was reported to enhance the secretion of factors mediating inflammatory and immune responses [13, 31]. Here, we found the mRNA expression of STC1, HGF, and TSG-6 was significantly increased in 3D-culured MSCs (Fig. 4A). The in vivo 


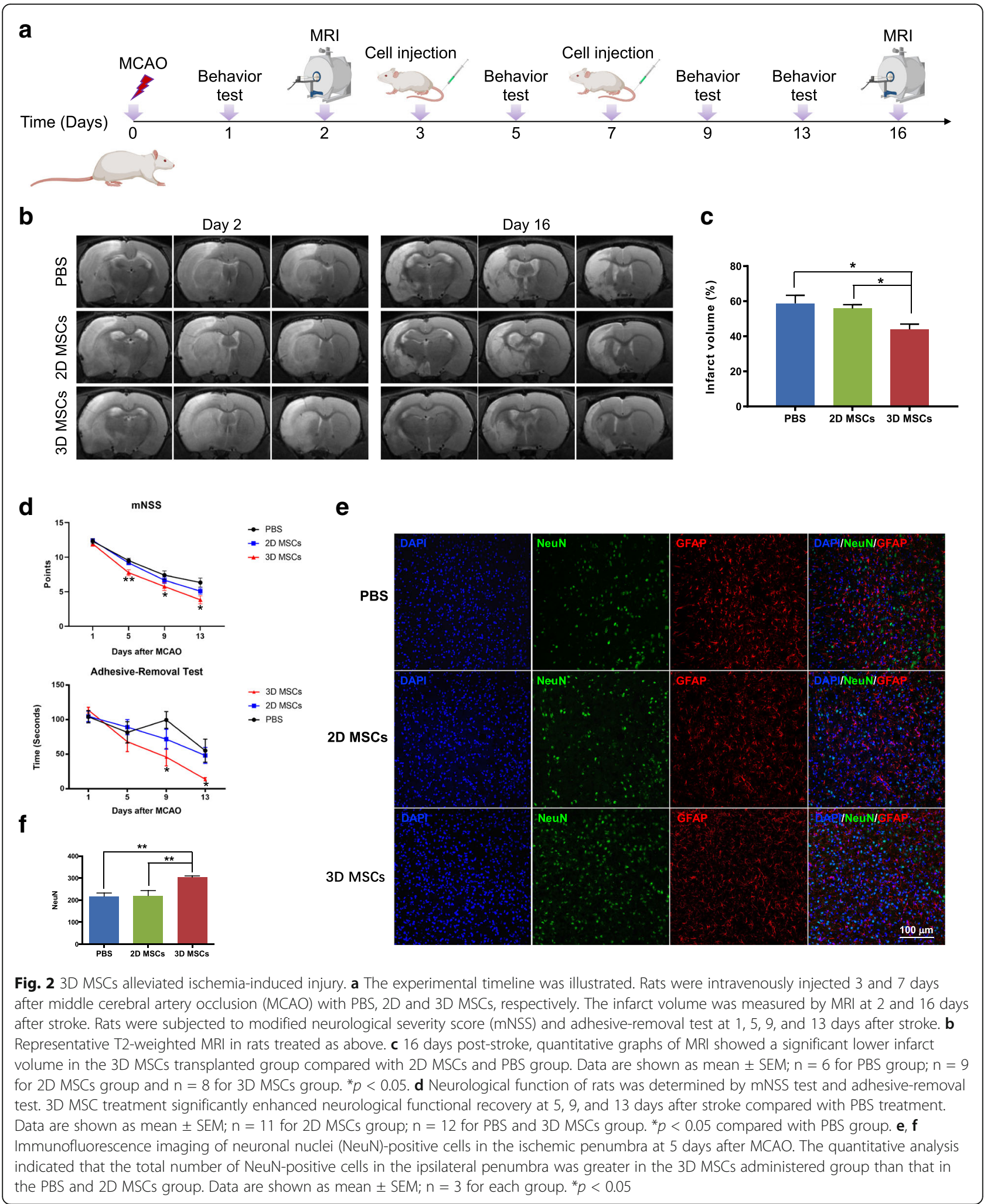

anti-inflammatory effects of 3D MSCs on microglia were further investigated with staining of microglia marker Iba1 in brain tissues at 2 days after cells infusion.
Astrocyte marker glial fibrillary acidic protein (GFAP) was also stained to indicate the ischemic core, as astrocytes form glial scar in the perilesional area after 


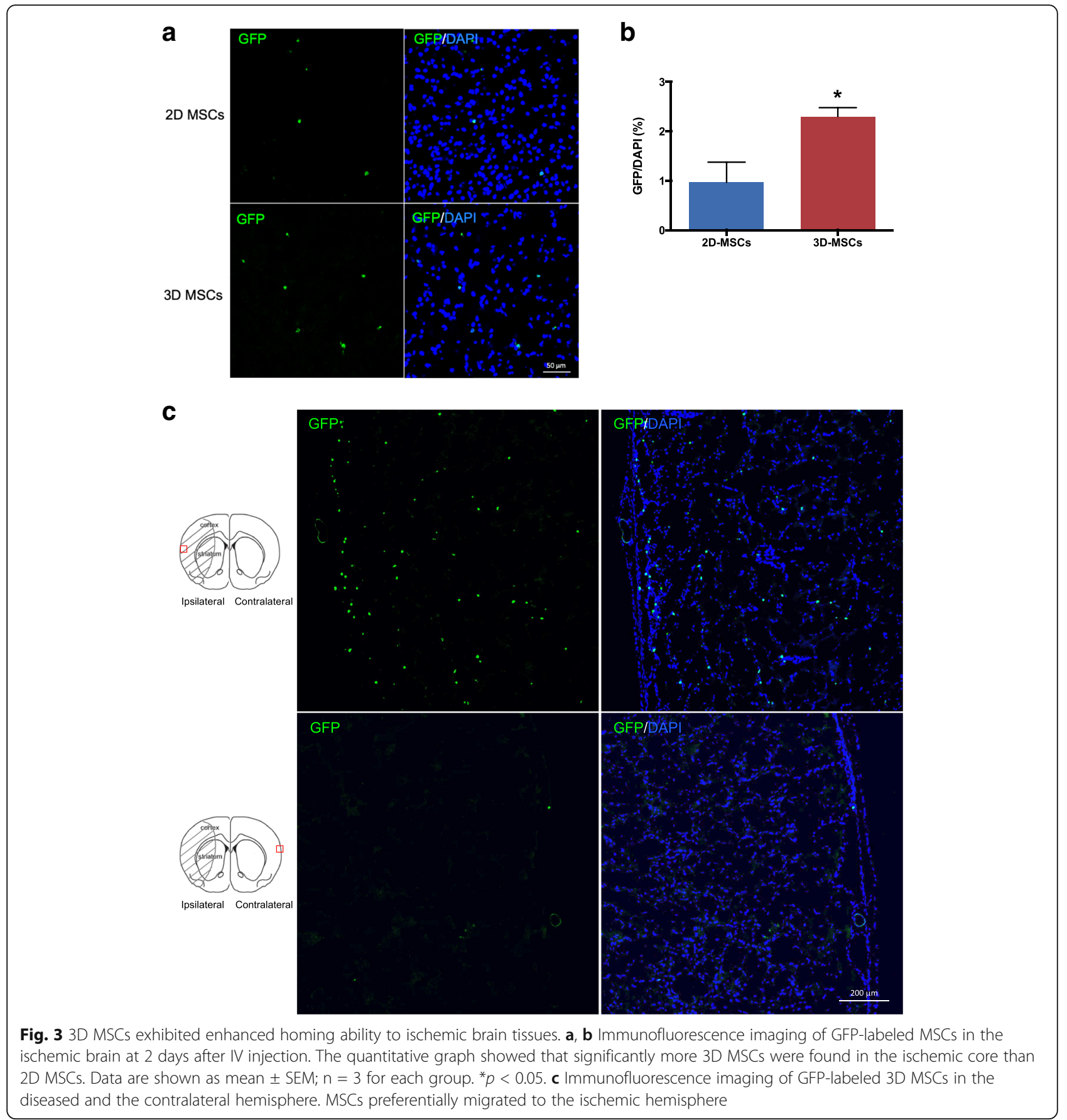

ischemia [32]. Iba1-positive microglia were observed in both the intact and the ischemic hemisphere with different morphological appearances. Ramified cells with long thin processes were mainly localized in the intact area of the ischemic hemisphere and the contralateral hemisphere. Intermediate cells with swollen processes were mostly observed in the peri-infarct regions. Amoeboid cells with round shape and no processes were mainly detected in the ischemic core [33] (Fig. 4B). When stimulated, microglia gained morphological changes from a ramified shape to an amoeboid-shaped "activation" status [34]. As amoeboid microglia were shown to be abundant in the core area between 3 and 7 days after stroke, we compared the number of amoeboid microglia 5 days after MCAO. The number of amoeboid microglia in the ischemic core was significantly decreased in the 3D MSCs group compared to the PBS and 2D MSCs group as shown by the immunofluorescence staining (Fig. 4C, D). qRT-PCR results also demonstrated decreased expression of microglial markers $I b a 1$ and $C D 45$ in 3D 





MSCs group (Fig. 4E). The expression of proinflamma-

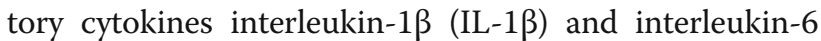
(IL-6) was further determined and results showed a reduction of their expression in 3D MSCs group than PBS and 2D MSCs group (Fig. 4F). These data suggest enhanced anti-inflammatory effects of 3D MSCs on microglia after ischemic injury.

\section{Transcriptomic changes in microglia with MSC administration}

To elucidate the molecular changes in microglia with MSCs transplantation, $\mathrm{CD} 45^{\text {low }} \mathrm{CD} 11 \mathrm{~b}^{+}$microglia were isolated 2 days after cell administration for RNA-seq analysis (Fig. 5A, Supplemental Fig. 1). The principal component analysis (PCA) showed that the transcriptome of microglia from sham-operated rats (Sham-MG) and 3D MSC-infused rats (3D-MG) differ from the transcriptome of microglia from PBS (PBS-MG) or 2D MSC (2D-MG)-treated rats (Fig. 5Ba). The Venn diagram showed the DEGs between Sham-MG with PBS-MG, 2D-MG, or 3D-MG, which demonstrated the high similarity of Sham-MG and 3D-MG (Fig. 5Bb). DEGs were processed for KEGG pathway enrichment analysis and results showed enrichment of 341 genes for immune system. Heatmap of the expression of these genes showed the clustering of Sham-MG and 3D-MG (Fig. 5Ca). The 530 downregulated genes in 3D-MG compared with PBS-MG and 2D-MG were subjected to Gene Ontology (GO) biological process analysis. Results revealed the involvement of genes in biological processes including cellular response to LPS, inflammatory responses, immune response, and immune system process (Fig. $5 \mathrm{Cb}$ ). Gene set enrichment analysis (GSEA) illustrated that immune response related genes were enriched among the DEGs in PBS-MG and 2D-MG (Fig. 5Cc). Among these genes, the expression of proinflammatory mediators including $I L-12 \beta, H m g b 1, C x c l 10, C c l 2$, and $I L-1 \beta$ were downregulated in 3D-MG (Fig. 5Cd).

Sterile inflammation leads to the release of damageassociated molecular patterns (DAMPs), which initiate inflammatory responses through activating DAMP receptors. Among all these DAMP receptors, macrophageinducible c-type lectin (Mincle) is the only one which is reported to be expressed by macrophages and plays a role in the pathogenesis after ischemic stroke $[35,36]$. Interestingly, we found a reduction in the expression of Mincle in 3D-MG (Fig. 5D). The downregulation of Mincle in 3D MSC-infused rats was validated with realtime RT-PCR (Fig. 5E). In order to further validate the effects of 3D MSCs on the expression of Mincle, the in vitro model using BV2 microglial cells was used. LPSactivated microglial cells were subjected with the conditioned media of 2D or 3D MSCs and the expression of Mincle was determined. Results also showed a significant decrease of Mincle expression in 3D MSC-treated microglia (Fig. 5F). These data suggest that Mincle may be involved in 3D MSC-mediated reduced activation of microglia after ischemic injury.

\section{Discussion}

MSCs are proposed as a promising therapy for treatment of ischemic stroke. Numerous preclinical and clinical studies support the non-invasive IV infusion of MSCs in stroke [7-9]. There is a general consensus that the homing and engraftment potential of cultured MSCs is lost after expanding under conventional culture conditions [37]. In this study, we investigated whether $3 \mathrm{D}$ culture condition can enhance the homing potential of MSCs. To this end, we transplanted MSCs in MCAO rats and results confirmed enhanced homing of 3D MSCs to the ischemic brain. This may due to the increased expression of chemokine receptor CXCR4 after 3D culture. CXCR4 encodes a well-known receptor for chemokine CXCL12, which is mainly produced by damaged neurons after cerebral ischemia [38]. The CXCL12/CXCR4 axis mediates homing of MSCs to the sites of injury [39].

After ischemic stroke, an inflammatory response is stimulated with raised proinflammatory cytokines and reduced anti-inflammatory and trophic factors [40]. The anti-inflammatory properties of MSCs in stroke therapy have been extensively mentioned. MSCs can promote the polarization of macrophages towards an antiinflammatory phenotype by producing immunosuppressive molecules and metabolites including prostaglandin E2 [41], TSG-6 [42], lactate [43], kynurenic acid [42], and spermidine [44]. MSCs can also prevent the infiltration of monocytes, macrophages, and neutrophils to inflammation sites through the production of TSG-6 [42, 45]. Moreover, MSCs can decrease markers of microglial activation (lower ED1 and Iba1) [21]. However, the underlying molecular mechanisms of MSCs in modulating microglia are not fully elucidated. To this end, we determined the transcriptomic changes of microglia with administration of 2D or 3D MSCs. RNA-seq results showed high similarity of the transcriptome from ShamMG and 3D-MG, indicating reduced microglial activation by 3D MSCs transplantation. GO biological process analysis implied involvement of downregulated genes in 3D-MG in biological processes including cellular response to LPS, inflammatory responses, immune response, and immune system process. GSEA also demonstrated enrichment of the DEGs in PBS-MG and 2D-MG in immune response. Moreover, the proinflammatory mediators, such as $I L-1 \beta, I L-12 \beta, H m g b 1, C x c l 10$, and $\mathrm{Ccl} 2$ were downregulated in $3 \mathrm{D}-\mathrm{MG}$. As for the mechanism of the anti-inflammatory effects of 3D MSCs on microglia, we determined the expression of antiinflammatory mediators STC1, HGF, and TSG-6. Results 
A

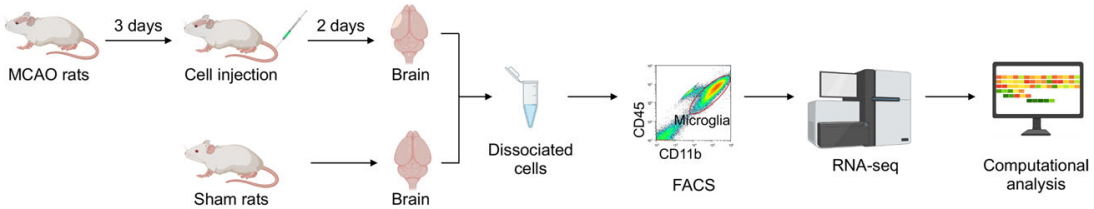

B (a)

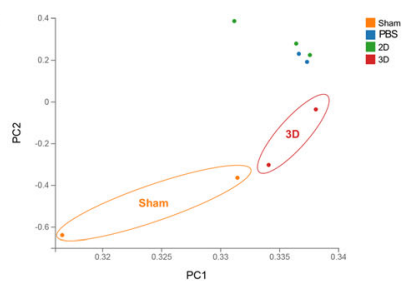

C

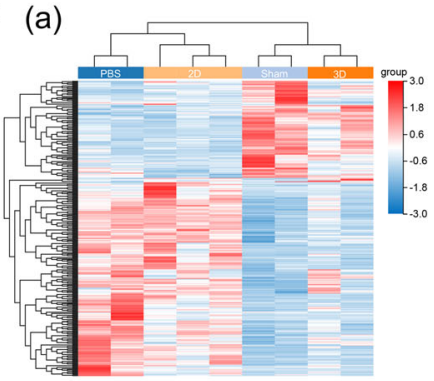

(c)

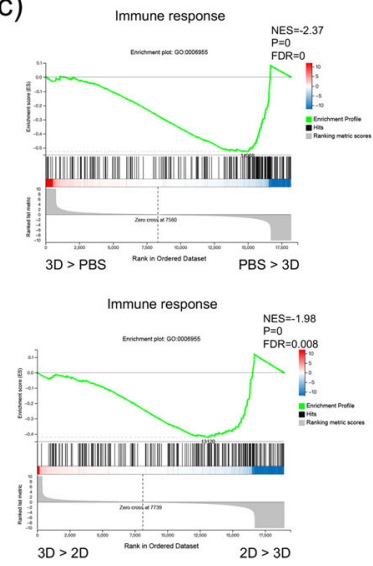

(b)

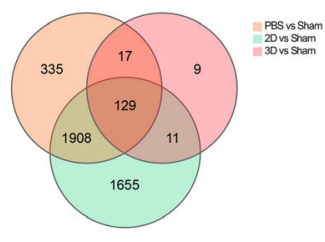

(b)

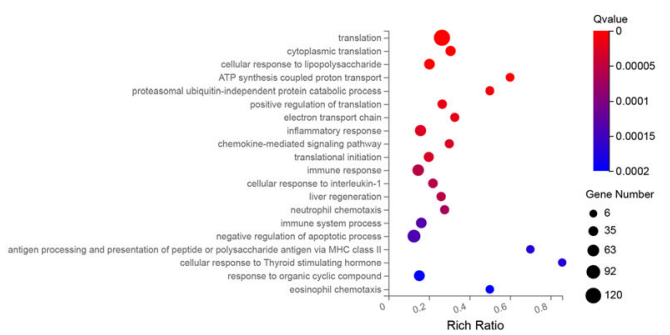

(d)
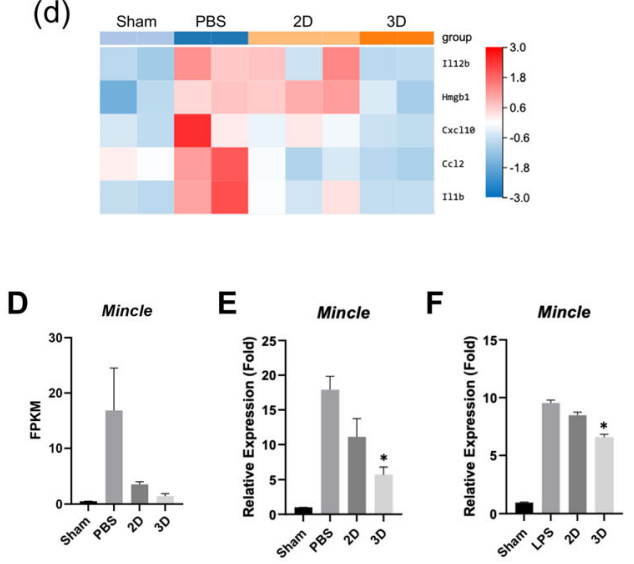

Fig. 5 Transcriptomic changes in microglia with MSC administration. A A schematic diagram depicting sample preparation for RNA-seq. $\mathrm{CD} 45^{\text {low }} \mathrm{CD} 11 \mathrm{~b}^{+}$microglia were isolated from the brain of Sham rats or the ischemic hemisphere 2 days after cell infusion. Total RNA was extracted from isolated microglia for RNA-seq analysis. B PCA (a) of microglia population sampled from sham rats, PBS-, 2D MSC-, Or 3D MSCtreated rats using PC1 and PC2 suggests clear separation of sham microglia and 3D MSC-treated microglia from PBS and 2D MSC-treated microglia. Venn diagram (b) showing the interactions among DEGs between Sham with PBS, 2D, or 3D MSC-treated samples. C Heatmap (a) showing the expression profiles of 341 genes which participate in the immune system. Gene Ontology (GO) biological process analysis (b) for the 530 downregulated genes by 3D MSC infusion compared with PBS and 2D MSC infusion showed genes involved in biological processes including cellular response to lipopolysaccharide, inflammatory responses, immune response, and immune system process. Gene set enrichment analysis (GSEA) (c) showing positive enrichment in transcriptome with treatment of PBS and 2D MSCs for immune response gene set. Heatmap (d) showing the expression profiles of genes belonging to proinflammatory mediators. D The expression of Mincle based on RNA-seq data. FPKM, fragments per kilobase of transcript per million mapped reads. E The expression of Mincle was validated with qRT-PCR. ${ }^{*} p<0.05$ compared with PBS group. $\mathbf{F}$ The expression of Mincle was determined in LPS-activated microglia with treatment of $2 \mathrm{D}$ or $3 \mathrm{D}$ MSCs conditioned media. ${ }^{*} p<0.05$ compared with LPS group 
showed significant increase of their expression in 3D MSCs. However, whether 3D MSCs exhibited antiinflammatory effects on microglia through secreting STC1, HGF, and TSG-6 need to be further investigated.

Sterile inflammation is stimulated by physical or chemical damage which leads to the release of damageassociated molecular patterns (DAMPs). DAMPs initiate an inflammatory response through the activation of pattern recognition receptors, including Toll-like receptors, NOD-like receptors, retinoic acid-inducible gene I-like receptors, C-type lectin receptors, and multiple intracellular DNA sensors [46]. Strikingly, we observed an attenuated expression of a DAMPs receptor, Mincle, in microglia derived from 3D MSCs injected rats. Mincle belongs to C-type lectin receptors and has been implicated in the development of inflammatory diseases. Mincle can induce proinflammatory responses in ischemic stroke model once binding Sin3A associated protein 130
(SAP130), a small ribonucleoprotein released by dying cells [36]. Mincle recognizes $\beta$-glucosylceramide, an intracellular metabolite released by damaged cells, and induced the production of proinflammatory cytokines by antigen-presenting cells [47]. The enhanced inflammation caused by the accumulation of $\beta$-glucosylceramide can be ameliorated by deletion of Mincle [47]. In our in vitro study, we observed a decreased expression of proinflammatory cytokines and Mincle with the treatment of the conditioned media from 3D MSCs. Our results suggest that decreased expression of Mincle in microglia mediated by 3D MSCs may be a cause of reduced inflammation in the injured tissue (Fig. 6).

\section{Conclusions}

In this study, we can conclude that 3D MSCs exhibited enhanced homing ability to the ischemic hemisphere. Moreover, improved therapeutic outcomes were

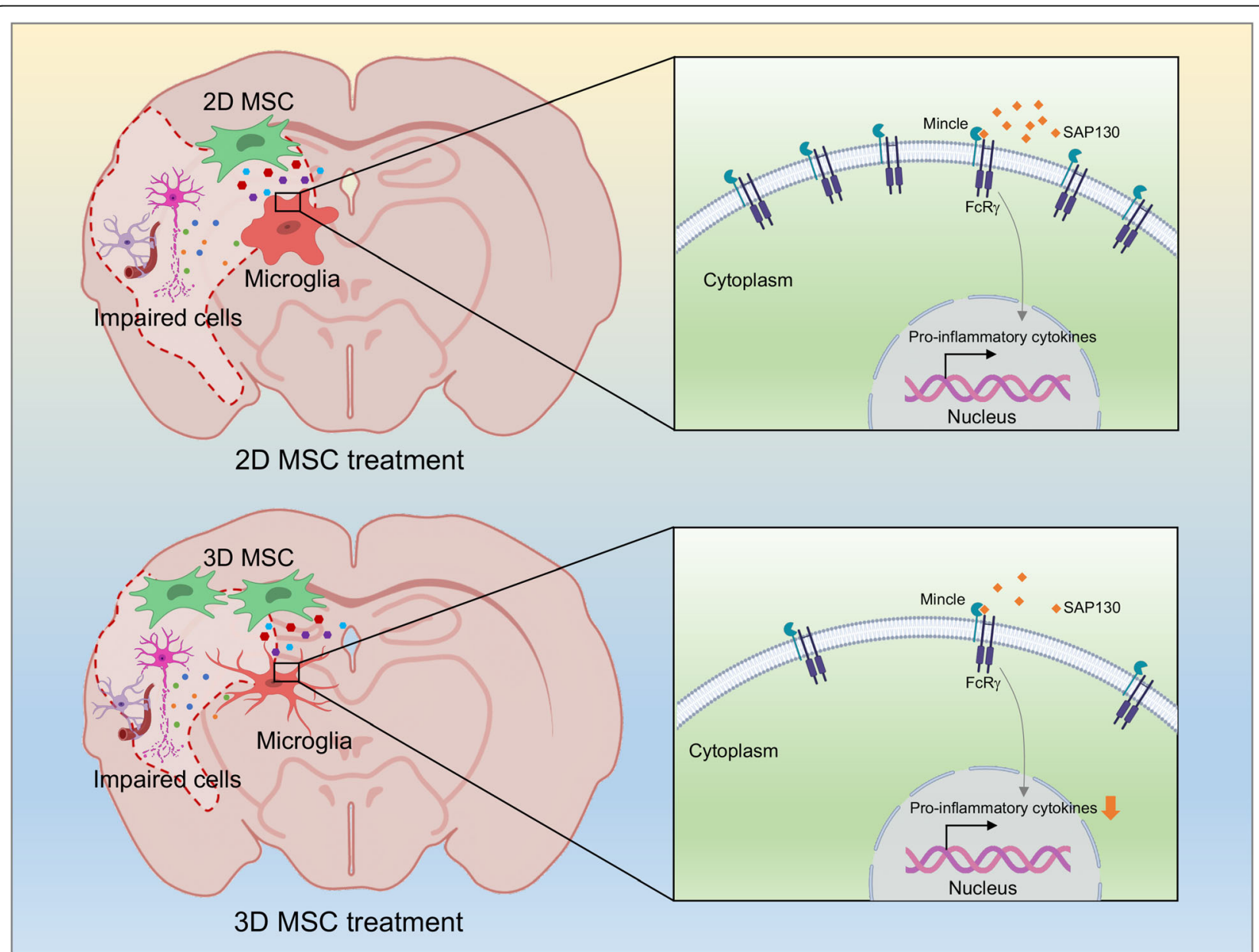

Fig. 6 A schematic diagram depicting a potential mechanism of 3D MSC-mediated repair to ischemic brain injury. Cells in the ischemic brain tissue release damage-associated molecular patterns (DAMPs) such as Sin3A associated protein 130 (SAP130), which bind to DAMP receptors such as Mincle on the surface of microglia, mediating increased production of proinflammatory cytokines by the cells. 3D MSCs exhibit enhanced homing and engraftment into the ischemic brain tissue following systemic administration, where they release mediators that decrease the expression of Mincle in microglia, thus reducing their production of proinflammatory cytokines 
observed in MCAO rats with 3D MSC administration. This may due to enhanced anti-inflammatory properties of 3D MSCs on microglia through reducing the expression of Mincle by microglia. In this sense, 3D MSCs could be exploited as a much safer and efficient alternative for the treatment of immune-mediated disorders.

\section{Abbreviations}

3D: Three-dimensional; MSCs: Mesenchymal stem cells; DAMPs: Damageassociated molecular patterns; IV: Intravenous; 2D: Two-dimensional; Iba1: Ionized calcium-binding adapter molecule 1; IL-6: Interleukin-6; TSG6: Tumor necrosis factor-stimulated gene-6; HGF: Hepatocyte growth factor: STC1: Stanniocalcin 1; MCAO: Middle cerebral artery occlusion; mNSS: Modified neurological severity score; DEGs: Differentially expressed genes; NeuN: Neuronal nuclei; GFAP: Glial fibrillary acidic protein; IL13: Interleukin-1 B; PCA: Principal component analysis; GO: Gene Ontology; LPS: Lipopolysaccharide; GSEA: Gene set enrichment analysis; SAP130: Sin3A associated protein 130; Mincle: Macrophage-inducible c-type lectin; FPKM: Fragments per kilobase of transcript per million mapped reads

\section{Supplementary Information}

The online version contains supplementary material available at https://doi. org/10.1186/s13287-021-02416-4.

\section{Additional file 1: Supplemental Figure 1. (A) Representative}

fluorescence-activated cell sorting (FACS) plots showing the population of microglia in brain tissue samples of different treatment groups. Dead cells were excluded with a dead cell marker 7-aminoactinomycin D (7AAD) and debris by size. (B) The bar graphs depicting the percentage of microglia in all cells. Data are shown as mean $\pm S E M ; n=3$ for 2D MSCs group, $n=2$ for Sham, PBS and 3D MSCs group. Supplemental Table S1 Primers for real-time RT-PCR

\section{Acknowledgements}

We thank Bing Yu and Bihua Shi for technical assistance in confocal analysis.

\section{Authors' contributions}

YJW, YJL, and YKD conceived and designed the experiments. YJL, YKD, and YR performed the experiments and analyzed the data. HD, SL, JDX, YNZ, MHM, BYW, WBH, YPC, and SYY provided the experimental technical support and assisted in completing the study at different stages. YJL drafted the manuscript. YJW finalized the paper. The authors read and approved the final manuscript.

\section{Funding}

This work was supported by grants from the Natural Science Foundation of China (31961160702, 81903239), National Key R\&D Program of China (2017YFC1103304), Shenzhen Science and Technology Innovation Committee (JCYJ20180307123901314, JCYJ20190809180217220), State Key Laboratory of Chemical Oncogenomics fund, and Tsinghua Shenzhen International Graduate School Overseas Cooperation Fund (HW2018006).

\section{Availability of data and materials}

The datasets used and/or analyzed during the current study are available from the corresponding author on reasonable request.

\section{Declarations}

Ethics approval and consent to participate

All experiments were performed according to standard protocols, in compliance with the Guide of the Animal Ethics Committee of Tsinghua Shenzhen International Graduate School.

\section{Consent for publication}

Not applicable.

\section{Competing interests}

The authors declare that they have no competing interests.

\section{Author details}

'State Key Laboratory of Chemical Oncogenomics, and Shenzhen Key Laboratory of Health Sciences and Technology, Tsinghua Shenzhen International Graduate School, Tsinghua University, Shenzhen 518055, China. ${ }^{2}$ School of Life Sciences, Tsinghua University, Beijing 100084, China. ${ }^{3}$ Department of Neurology, The Chinese PLA General Hospital, Medical School of Chinese PLA, Beijing 100853, China. ${ }^{4}$ Tsinghua-Berkeley Shenzhen Institute (TBSI), Tsinghua University, Shenzhen 518055, China. ${ }^{5}$ Peking University Shenzhen Hospital, Shenzhen 518036, China. Institute of Antibody Engineering, School of Laboratory Medicine and Biotechnology, Southern Medical University, Guangzhou 510515, China. ${ }^{7}$ Department of Neurology, The Chinese PLA General Hospital, Beijing 100853, China.

Received: 17 March 2021 Accepted: 25 May 2021

Published online: 21 June 2021

\section{References}

1. Zhou M, Wang H, Zeng X, Yin P, Zhu J, Chen W, et al. Mortality, morbidity, and risk factors in china and its provinces, 1990-2017: a systematic analysis for the global burden of disease study 2017. Lancet. 2019:394(10204):114558. https://doi.org/10.1016/S0140-6736(19)30427-1.

2. Prabhakaran S, Ruff I, Bernstein RA. Acute stroke intervention: a systematic review. JAMA. 2015;313(14):1451-62. https://doi.org/10.1001/jama.2015.3058.

3. Gutierrez-Fernandez M, Rodriguez-Frutos B, Otero-Ortega L, Ramos-Cejudo fo Fuentes B, Diez-Tejedor E. Adipose tissue-derived stem cells in stroke treatment: from bench to bedside. Discov Med. 2013;16(86):37-43.

4. Patrikoski M, Sivula J, Huhtala H, Helminen M, Salo F, Mannerstrom B, et al. Different culture conditions modulate the immunological properties of adipose stem cells. Stem Cells Transl Med. 2014;3(10):1220-30. https://doi. org/10.5966/sctm.2013-0201.

5. Ra JC, Shin IS, Kim SH, Kang SK, Kang BC, Lee HY, et al. Safety of intravenous infusion of human adipose tissue-derived mesenchymal stem cells in animals and humans. Stem Cells Dev. 2011;20(8):1297-308. https://doi.org/1 0.1089/scd.2010.0466.

6. Cui L, Moisan A, Jolkkonen J. Intravascular cell therapy in stroke: predicting the future trends. Regen Med. 2019;14(1):63-8. https://doi.org/10.2217/ rme-2018-0037.

7. Caplan Al, Correa D. The msc: an injury drugstore. Cell Stem Cell. 2011;9(1): 11-5. https://doi.org/10.1016/j.stem.2011.06.008.

8. Caplan Al, Dennis JE. Mesenchymal stem cells as trophic mediators. J Cell Biochem. 2006;98(5):1076-84. https://doi.org/10.1002/jcb.20886.

9. Shi Y, Wang Y, Li Q, Liu K, Hou J, Shao C, et al. Immunoregulatory mechanisms of mesenchymal stem and stromal cells in inflammatory diseases. Nat Rev Nephrol. 2018;14(8):493-507. https://doi.org/10.1038/s41 581-018-0023-5.

10. Yong KW, Choi JR, Dolbashid AS, Wan Safwani WKZ. Biosafety and bioefficacy assessment of human mesenchymal stem cells: what do we know so far? Regen Med. 2018;13(2):219-32. https://doi.org/10.2217/rme-2 017-0078

11. Ge J, Guo L, Wang S, Zhang Y, Cai T, Zhao RC, et al. The size of mesenchymal stem cells is a significant cause of vascular obstructions and stroke. Stem Cell Rev Rep. 2014;10(2):295-303. https://doi.org/10.1007/s1201 5-013-9492-X.

12. Zhou Y, Chen $\mathrm{H}, \mathrm{Li} H, \mathrm{Wu}$ Y. 3d culture increases pluripotent gene expression in mesenchymal stem cells through relaxation of cytoskeleton tension. J Cell Mol Med. 2017;21(6):1073-84. https://doi.org/10.1111/ jcmm.12946.

13. Bartosh TJ, Ylostalo JH, Mohammadipoor A, Bazhanov N, Coble K, Claypool $\mathrm{K}$, et al. Aggregation of human mesenchymal stromal cells (mscs) into 3d spheroids enhances their antiinflammatory properties. Proc Natl Acad Sci USA. 2010;107(31):13724-9. https://doi.org/10.1073/pnas.1008117107.

14. Mo M, Zhou Y, Li S, Wu Y. Three-dimensional culture reduces cell size by increasing vesicle excretion. Stem Cells. 2018;36(2):286-92. https://doi.org/1 $0.1002 /$ stem.2729.

15. Wang $S$, Guo L, Ge J, Yu L, Cai T, Tian R, et al. Excess integrins cause lung entrapment of mesenchymal stem cells. Stem Cells. 2015;33(11):3315-26. https://doi.org/10.1002/stem.2087.

16. Shi M, Li J, Liao L, Chen B, Li B, Chen L, et al. Regulation of cxcr4 expression in human mesenchymal stem cells by cytokine treatment: role in homing efficiency in nod/scid mice. Haematologica. 2007:92(7):897-904. https://doi. org/10.3324/haematol.10669. 
17. Zhou P, Liu Z, Li X, Zhang B, Wang X, Lan J, et al. Migration ability and tolllike receptor expression of human mesenchymal stem cells improves significantly after three-dimensional culture. Biochem Biophys Res Commun. 2017;491(2):323-8. https://doi.org/10.1016/j.bbrc.2017.07.102.

18. Potapova IA, Brink PR, Cohen IS, Doronin SV. Culturing of human mesenchymal stem cells as three-dimensional aggregates induces functional expression of cxcr4 that regulates adhesion to endothelial cells. J Biol Chem. 2008;283(19):13100-7. https://doi.org/10.1074/jbc.M800184200.

19. Jin R, Yang G, Li G. Inflammatory mechanisms in ischemic stroke: role of inflammatory cells. J Leukoc Biol. 2010;87(5):779-89. https://doi.org/10.1189/ jlb.1109766.

20. Qin C, Zhou LQ, Ma XT, Hu ZW, Yang S, Chen M, et al. Dual functions of microglia in ischemic stroke. Neurosci Bull. 2019;35(5):921-33. https://doi. org/10.1007/s12264-019-00388-3.

21. McGuckin CP, Jurga M, Miller AM, Sarnowska A, Wiedner M, Boyle NT, et al. Ischemic brain injury: a consortium analysis of key factors involved in mesenchymal stem cell-mediated inflammatory reduction. Arch Biochem Biophys. 2013;534(1-2):88-97. https://doi.org/10.1016/j.abb.2013.02.005.

22. Zimmermann JA, McDevitt TC. Pre-conditioning mesenchymal stromal cell spheroids for immunomodulatory paracrine factor secretion. Cytotherapy. 2014:16(3):331-45. https://doi.org/10.1016/j.jcyt.2013.09.004.

23. Ylostalo JH, Bartosh TJ, Coble K, Prockop DJ. Human mesenchymal stem/ stromal cells cultured as spheroids are self-activated to produce prostaglandin e2 that directs stimulated macrophages into an antiinflammatory phenotype. Stem Cells. 2012;30(10):2283-96. https://doi.org/1 0.1002/stem.1191.

24. Dong G, Wang S, Ge Y, Deng Q, Cao Q, Wang Q, et al. Serum-free culture system for spontaneous human mesenchymal stem cell spheroid formation. Stem Cells Int. 2019;2019:6041816.

25. Santos JM, Camoes SP, Filipe E, Cipriano M, Barcia RN, Filipe M, et al. Threedimensional spheroid cell culture of umbilical cord tissue-derived mesenchymal stromal cells leads to enhanced paracrine induction of wound healing. Stem Cell Res Ther. 2015;6(1):90. https://doi.org/10.1186/ s13287-015-0082-5.

26. Cao J, Wang B, Tang T, Lv L, Ding Z, Li Z, et al. Three-dimensional culture of mscs produces exosomes with improved yield and enhanced therapeutic efficacy for cisplatin-induced acute kidney injury. Stem Cell Res Ther. 2020; 11(1):206. https://doi.org/10.1186/s13287-020-01719-2.

27. Guo L, Ge J, Zhou Y, Wang S, Zhao RC, Wu Y. Three-dimensional spheroidcultured mesenchymal stem cells devoid of embolism attenuate brain stroke injury after intra-arterial injection. Stem Cells Dev. 2014;23(9):978-89. https://doi.org/10.1089/scd.2013.0338.

28. Li Z, Liu C, Xie Z, Song P, Zhao RC, Guo L, et al. Epigenetic dysregulation in mesenchymal stem cell aging and spontaneous differentiation. Plos One. 2011;6(6):e20526. https://doi.org/10.1371/journal.pone.0020526.

29. Boltze J, Kowalski I, Geiger K, Reich D, Gunther A, Buhrle C, et al Experimental treatment of stroke in spontaneously hypertensive rats by cd34+ and cd34- cord blood cells. Ger Med Sci. 2005;3:Doc09.

30. Chen J, Li Y, Wang L, Zhang Z, Lu D, Lu M, et al. Therapeutic benefit of intravenous administration of bone marrow stromal cells after cerebral ischemia in rats. Stroke. 2001;32(4):1005-11. https://doi.org/10.1161/01. STR.32.4.1005.

31. Bartosh TJ, Ylostalo JH, Bazhanov N, Kuhlman J, Prockop DJ. Dynamic compaction of human mesenchymal stem/precursor cells into spheres selfactivates caspase-dependent il 1 signaling to enhance secretion of modulators of inflammation and immunity (pge2, tsg6, and stc1). Stem Cells. 2013;31(11):2443-56. https://doi.org/10.1002/stem.1499.

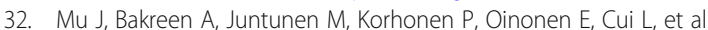
Combined adipose tissue-derived mesenchymal stem cell therapy and rehabilitation in experimental stroke. Front Neurol. 2019;10:235. https://doi. org/10.3389/fneur.2019.00235.

33. Hamzei Taj S, Le Blon D, Hoornaert C, Daans J, Quarta A, Praet J, et al. Targeted intracerebral delivery of the anti-inflammatory cytokine il13 promotes alternative activation of both microglia and macrophages after stroke. J Neuroinflammation. 2018;15(1):174. https://doi.org/10.1186/s12974018-1212-7.

34. Li Q, Barres BA. Microglia and macrophages in brain homeostasis and disease. Nat Rev Immunol. 2018:18(4):225-42. https://doi.org/10.1038/nri.2017.125.

35. Gong T, Liu L, Jiang W, Zhou R. Damp-sensing receptors in sterile inflammation and inflammatory diseases. Nat Rev Immunol. 2020;20(2):95112. https://doi.org/10.1038/s41577-019-0215-7.
36. Suzuki Y, Nakano Y, Mishiro K, Takagi T, Tsuruma K, Nakamura M, et al. Involvement of mincle and syk in the changes to innate immunity after ischemic stroke. Sci Rep. 2013;3(1):3177. https://doi.org/10.1038/srep03177.

37. Rombouts WJ, Ploemacher RE. Primary murine msc show highly efficient homing to the bone marrow but lose homing ability following culture. Leukemia. 2003;17(1):160-70. https://doi.org/10.1038/sj.leu.2402763.

38. Ruscher K, Kuric E, Liu Y, Walter HL, Issazadeh-Navikas S, Englund E, et al. Inhibition of $\mathrm{cxCl} 12$ signaling attenuates the postischemic immune response and improves functional recovery after stroke. J Cereb Blood Flow Metab. 2013;33(8):1225-34. https://doi.org/10.1038/jcbfm.2013.71.

39. Hocking AM. The role of chemokines in mesenchymal stem cell homing to wounds. Adv Wound Care (New Rochelle). 2015;4(11):623-30. https://doi. org/10.1089/wound.2014.0579.

40. Pei J, You X, Fu Q. Inflammation in the pathogenesis of ischemic stroke. Front Biosci. 2015:20:772-83.

41. Vasandan AB, Jahnavi S, Shashank C, Prasad P, Kumar A, Prasanna SJ. Human mesenchymal stem cells program macrophage plasticity by altering their metabolic status via a pge2-dependent mechanism. Sci Rep. 2016;6(1): 38308. https://doi.org/10.1038/srep38308.

42. Wang G, Cao K, Liu K, Xue Y, Roberts Al, Li F, et al. Kynurenic acid, an ido metabolite, controls tsg-6-mediated immunosuppression of human mesenchymal stem cells. Cell Death Differ. 2018;25(7):1209-23. https://doi. org/10.1038/s41418-017-0006-2.

43. Selleri S, Bifsha P, Civini S, Pacelli C, Dieng MM, Lemieux W, et al. Human mesenchymal stromal cell-secreted lactate induces m2-macrophage differentiation by metabolic reprogramming. Oncotarget. 2016;7(21):30193210. https://doi.org/10.18632/oncotarget.8623.

44. Yang Q, Zheng C, Cao J, Cao G, Shou P, Lin L, et al. Spermidine alleviates experimental autoimmune encephalomyelitis through inducing inhibitory macrophages. Cell Death Differ. 2016;23(11):1850-61. https://doi.org/10.103 8/cdd.2016.71.

45. Song HB, Park SY, Ko JH, Park JW, Yoon CH, Kim DH, et al. Mesenchymal stromal cells inhibit inflammatory lymphangiogenesis in the cornea by suppressing macrophage in a tsg-6-dependent manner. Mol Ther. 2018; 26(1):162-72. https://doi.org/10.1016/j.ymthe.2017.09.026.

46. Cao X. Self-regulation and cross-regulation of pattern-recognition receptor signalling in health and disease. Nat Rev Immunol. 2016;16(1):35-50. https:// doi.org/10.1038/nri.2015.8.

47. Nagata M, Izumi Y, Ishikawa E, Kiyotake R, Doi R, Iwai S, et al. Intracellular metabolite beta-glucosylceramide is an endogenous mincle ligand possessing immunostimulatory activity. Proc Natl Acad Sci USA. 2017; 114(16):E3285-E94. https://doi.org/10.1073/pnas.1618133114.

\section{Publisher's Note}

Springer Nature remains neutral with regard to jurisdictional claims in published maps and institutional affiliations.
Ready to submit your research? Choose BMC and benefit from:
- fast, convenient online submission
- thorough peer review by experienced researchers in your field
- rapid publication on acceptance
- support for research data, including large and complex data types
- gold Open Access which fosters wider collaboration and increased citations
- maximum visibility for your research: over $100 \mathrm{M}$ website views per year
At BMC, research is always in progress.
Learn more biomedcentral.com/submissions 